\title{
PROPRIEDADES MECÂNICAS A ALTAS TEMPERATURAS DE FERROS FUNDIDOS CINZENTOS APLICADOS A CABEÇOTES DE MOTOR A DIESEL*
}

\section{Resumo}

Heloíza Döering Gasparin ${ }^{1}$ Guilherme Ourique Verran² Wilson Luiz Guesser ${ }^{3}$ Masahiro Tomiyama ${ }^{4}$ Carlos Eduardo Junges ${ }^{5}$

Os efeitos de elevadas temperaturas e do tempo de exposição sobre as propriedades mecânicas de um ferro fundido cinzento hipoeutético foram avaliados em amostras de dois ferros fundidos da classe FC300, de mesma base, com diferentes percentuais de molibdênio. Ensaios de tração até ruptura a quente foram realizados em corpos de prova com diâmetro de $10 \mathrm{~mm}$, usinados de um pino bruto de $30 \mathrm{~mm}$ de diâmetro. Os ensaios foram realizados a frio (temperatura ambiente) e a quente $\left(200^{\circ} \mathrm{C}, 300^{\circ} \mathrm{C}\right.$, $350^{\circ} \mathrm{C}, 400^{\circ} \mathrm{C}, 450^{\circ} \mathrm{C}, 500^{\circ} \mathrm{C}$ e $550^{\circ} \mathrm{C}$ ). Os tempos de exposição a elevadas temperaturas, após homogeneização térmica do material, foram de $0,60,90$ e 120 minutos. As amostras com maior percentual de molibdênio $(0,27 \%$ em peso) apresentaram melhores propriedades mecânicas para todas as condições ensaiadas. De acordo com a literatura a redução na resistência mecânica com a elevação da temperatura é atribuída à ocorrência de decomposição da perlita, como resultado da difusão do carbono vindo da cementita às lamelas de grafita, que é o responsável pelo aumento da quantidade de grafita no material. As análises metalográficas evidenciam a ocorrência deste fenômeno. Aumentos no tempo de exposição a temperaturas elevadas também contribuíram para a degeneração da perlita.

Palavras-chave: Ferro fundido cinzento; Perlita; Temperaturas elevadas; Propriedades mecânicas.

\section{GRAY CASTING IRONS MECHANICAL PROPERTIES AT ELEVATED TEMPERATURE FOR CYLINDER HEAD APPLICATIONS}

\section{Abstract}

The effects of high temperatures and exposure time on the mechanical properties of a hypoeutectic gray cast iron were evaluate in two samples of cast iron FC300 class, with different percentages of molybdenum. Elevated temperature stress-rupture tests were perform on $10 \mathrm{~mm}$ diameter specimens, machined from a $30 \mathrm{~mm}$ diameter pin. Assays were perform at room temperature and high temperature $\left(200^{\circ} \mathrm{C}, 300^{\circ} \mathrm{C}, 350^{\circ} \mathrm{C}, 400^{\circ} \mathrm{C}, 450^{\circ} \mathrm{C}\right.$, $500^{\circ} \mathrm{C}$ and $550^{\circ} \mathrm{C}$ ). The exposure times at elevated temperatures, after material thermal homogenization, were 0, 60, 90 and 120 minutes. Samples with higher percentage of molybdenum (0.27 wt\%) resulted in better mechanical properties in all tested conditions. According to the literature, increasing the temperature, the tensile strength fall by the occurrence of perlite breakdown, where some carbon migrate to the graphite lamellae, which is responsible for increasing the graphite amount in the material. The metallographic analysis reveals the occurrence of this phenomenon. Increases in exposure time at elevated temperatures also contributed to the perlite degeneration.

Keywords: Gray cast iron; Perlite; High temperature; Mechanical properties.

1 Engenheira mecânica, mestranda do Programa de Pós-Graduação em Ciência e Engenharia de Materiais (PGCEM), Universidade do Estado de Santa Catarina (UDESC), Joinville, Santa Catarina, Brasil.

2 Dr. Eng., Professor do Departamento de Engenharia Mecânica (DEM) e do PGCEM, Universidade do Estado de Santa Catarina (UDESC), Joinville, Santa Catarina, Brasil.

3 Dr. Eng., Professor, DEM e PGCEM, UDESC, Diretor da Tupy S.A., Joinville, Santa Catarina, Brasil.

4 Dr. Eng., Professor, DEM e PGCEM, UDESC, Joinville, Santa Catarina, Brasil.

5 Acadêmico de Engenharia Mecânica, UDESC, Joinville, Santa Catarina, Brasil.

* Contribuição técnica ao $69^{\circ}$ Congresso Anual da ABM - Internacional e ao 14ํㅡㄹ ENEMET - Encontro Nacional de Estudantes de Engenharia Metalúrgica, de Materiais e de Minas, 21 a 25 de julho de 2014, São Paulo, SP, Brasil. 


\section{INTRODUÇÃO}

O desenvolvimento e o investimento em pesquisas de materiais utilizados em cabeçotes de motor a diesel é justificado pela necessidade de maiores performances e desempenhos. Nechtelberger [1] aponta como um dos maiores desafios dentro deste contexto a obtenção de materiais com elevada resistência mecânica quando submetidos a elevadas temperaturas de trabalho. Mesmo que este tema já tenha sido estudado por diversos autores [1-5], até o presente momento não existe um consenso sobre os mecanismos atuantes sobre o comportamento mecânico dos ferros fundidos cinzentos a elevadas temperaturas.

A comparação de ferros fundidos cinzentos perlíticos com diferentes teores de molibdênio ( $\mathrm{A} 0$ e $\mathrm{A} 1$ ) foi realizada através de ensaios mecânicos de tração até a ruptura, desde a temperatura ambiente até $550^{\circ} \mathrm{C}$, uma vez que cabeçotes de motor a diesel estão sujeitos a temperatura máxima de trabalho de $540^{\circ} \mathrm{C}$ [1]. Com a análise das propriedades que foram obtidas a partir da curva "tensão x deformação", observou-se o comportamento do material quando submetido a determinadas temperaturas. Análises metalográficas e químicas também foram utilizadas no sentido obter evidências sobre os fenômenos que determinaram estas variações nas propriedades mecânicas em função da temperatura de ensaio.

Adicionalmente, a pesquisa contempla a avaliação do tempo de exposição a elevadas temperaturas. Cada material teve seu conjunto de propriedades mecânicas a quente estudado em tempo de exposição padrão (tempo necessário apenas para homogeneização da temperatura do material, 30min, e também com tempos de exposição estendidos além da homogeneização térmica do material (60min, 90min e 120min). Com a variação de tempos de exposição a altas temperaturas, pretendeuse aprofundar o entendimento da estabilidade microestrutural dos materiais, que é um ponto fundamental para a aplicação.

A microestrutura do ferro fundido cinzento perlítico de baixa liga, geralmente é composta por veios de grafita que formam um esqueleto contínuo nas células eutéticas, matriz perlítica e um determinado percentual de carbonetos [6]. A formação de carbonetos está relacionada com o teor de elementos de liga adicionados ao material e também com a velocidade de resfriamento [7]. A estabilidade microestrutural de ferros fundidos cinzentos perlíticos, é significativamente afetada pelo aumento de temperatura, o que pode resultar diretamente na degradação das propriedades deste material [8].

Elementos de liga como $\mathrm{Cr}$ e Mo inibem a velocidade da decomposição da perlita em ferros fundidos cinzentos expostos à elevada temperatura [2]. O molibdênio, um dos elementos mais influentes para o aumento da resistência mecânica e dureza a quente, possui tendência ao coquilhamento bem inferior que outros elementos estabilizadores de carbonetos. Normalmente utilizado junto com outros elementos estabilizadores de carbonetos, o molibdênio age como um forte refinador e estabilizador da perlita, o que dificulta transformações na microestrutura [9].

Para a maioria das aplicações a elevadas temperaturas, até $300^{\circ} \mathrm{C}$ os materiais apresentam boa rigidez. Para temperaturas superiores a $300^{\circ} \mathrm{C}$, a resistência à tração começa a diminuir ligeiramente. Este comportamento acentua-se notadamente para temperaturas superiores a $400^{\circ} \mathrm{C}$ [2]. Palmer [10] considera que acima de $350^{\circ} \mathrm{C}$, ferros fundidos ligados são altamente recomendados, pois a partir deste ponto a temperatura pode tornar a estrutura da cementita ainda mais instável. Sabe-se que praticamente todos os ferros fundidos apresentam quedas bruscas da resistência à tração em torno de $400^{\circ} \mathrm{C}$. Quando este material é ligado ao

* Contribuição técnica ao $69^{\circ}$ Congresso Anual da ABM - Internacional e ao 14ํㅡㄹ ENEMET - Encontro Nacional de Estudantes de Engenharia Metalúrgica, de Materiais e de Minas, 21 a 25 de julho de 2014, São Paulo, SP, Brasil. 


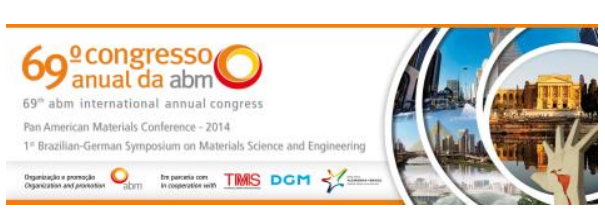

Evidencia-se a influência da adição de elementos de liga na retenção de boas propriedades mecânicas a elevadas temperaturas, principalmente da combinação dos elementos de liga cromo e molibdênio [16], Estes elementos de liga também são conhecidos por retardarem o processo de decomposição da perlita a elevadas temperaturas para ferros fundidos cinzentos [2].

A figura 2 apresenta o resultado de ensaios de tração até ruptura a elevadas temperaturas para um ferro fundido cinzento classe FC250, que mostra a queda brusca da resistência à tração acima de $400^{\circ} \mathrm{C}$, devido à ocorrência de decomposição da perlita [13].

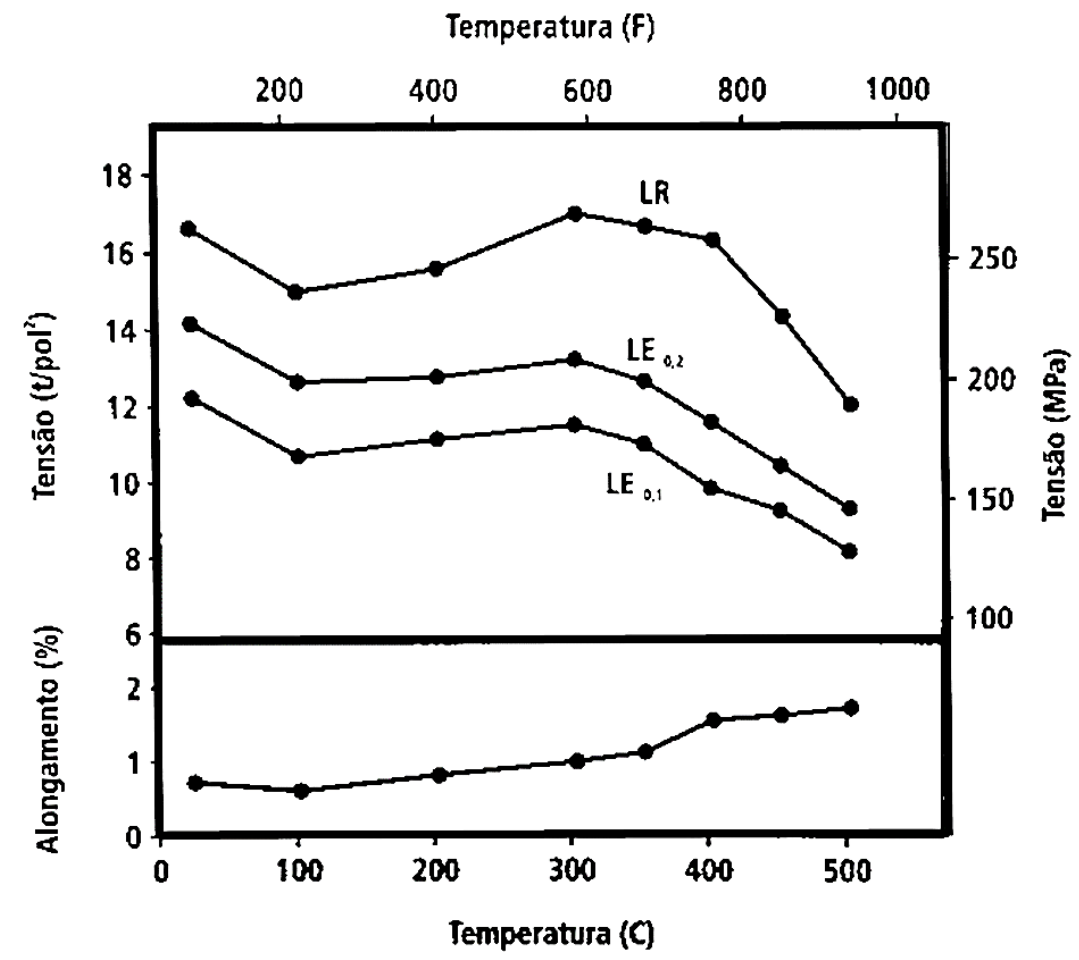

Figura 2. Variação de propriedades mecânicas com a temperatura para um ferro fundido cinzento classe FC250 [13].

A temperatura ambiente o material exibe limite de resistência à tração de $268 \mathrm{MPa}$. Röhrig [17] comenta que a redução desta propriedade, entre $150^{\circ} \mathrm{C}$ e $200^{\circ} \mathrm{C}$, está relacionada a tensões geradas pela dilatação da cementita.

Acima de $400^{\circ} \mathrm{C}$ a resistência apresenta maiores reduções em função da instabilidade microestrutural, conforme descrito anteriormente [10]. O aumento da resistência a quente seria possível com a adição de elementos de liga como $\mathrm{Mo}, \mathrm{Ni}$, Sn, Cr e Cu, que são os mais utilizados para estas condições [8].

\section{MATERIAIS E MÉTODOS}

O material base utilizado neste estudo foi um ferro fundido cinzento perlítico ligado, sendo que a diferença entre os dois materiais avaliados está no percentual de molibdênio presente em cada um deles (tabela 2). Ainda elementos de liga como Cromo, Cobre e Estanho estão presentes nesta liga.

\footnotetext{
* Contribuição técnica ao 69ำ Congresso Anual da ABM - Internacional e ao 14ํㅡㄹ ENEMET - Encontro Nacional de Estudantes de Engenharia Metalúrgica, de Materiais e de Minas, 21 a 25 de julho de 2014, São Paulo, SP, Brasil.
} 


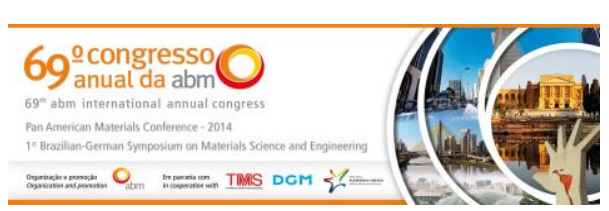

diferença média de $28 \mathrm{MPa}(9 \%)$ nesta propriedade à temperatura ambiente, e $32 \mathrm{MPa}(14 \%)$ a $400^{\circ} \mathrm{C}$ como mostrado na figura 5 . O aumento percentual médio do limite de resistência à tração, causado por $0,06 \%$ de Molibdênio, ficou em torno de $11 \%$.

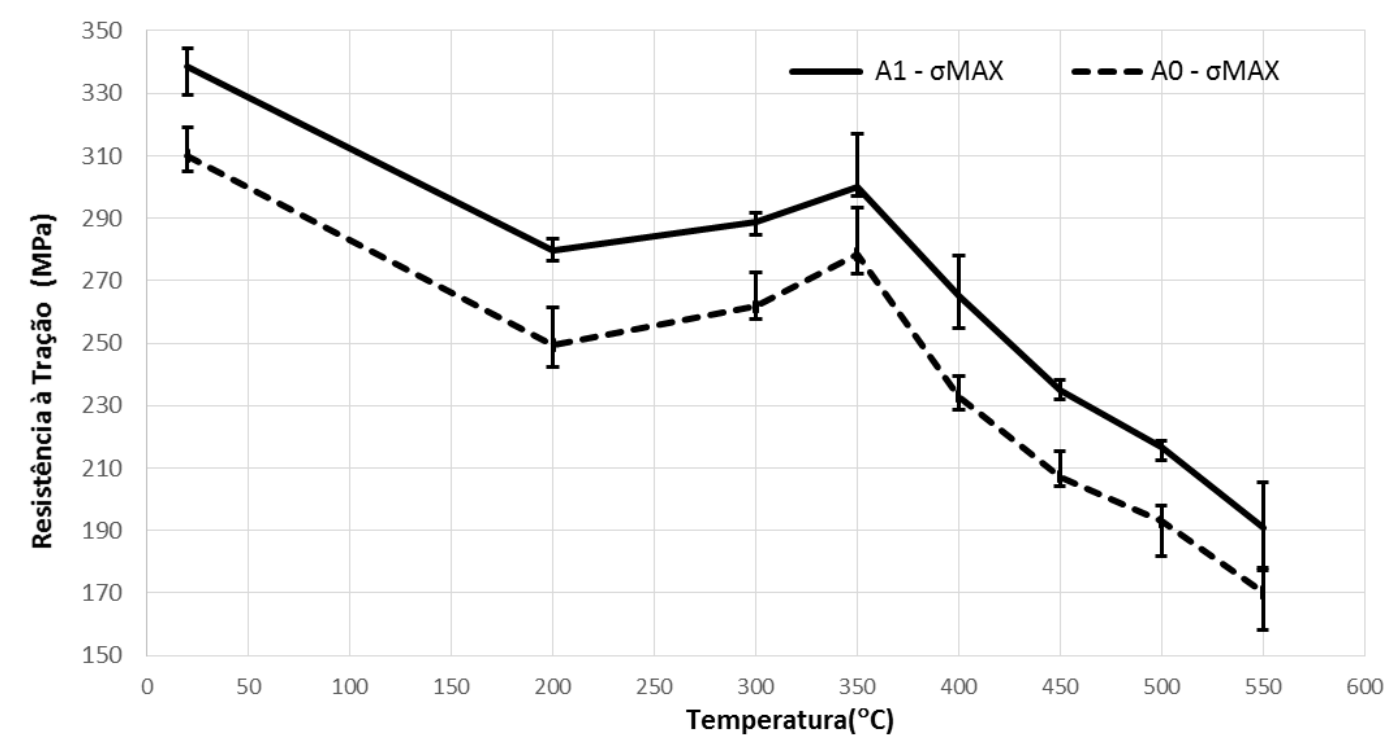

Figura 4. Variação do limite máximo de resistência à tração em função da temperatura de ensaio para as duas composições estudadas.

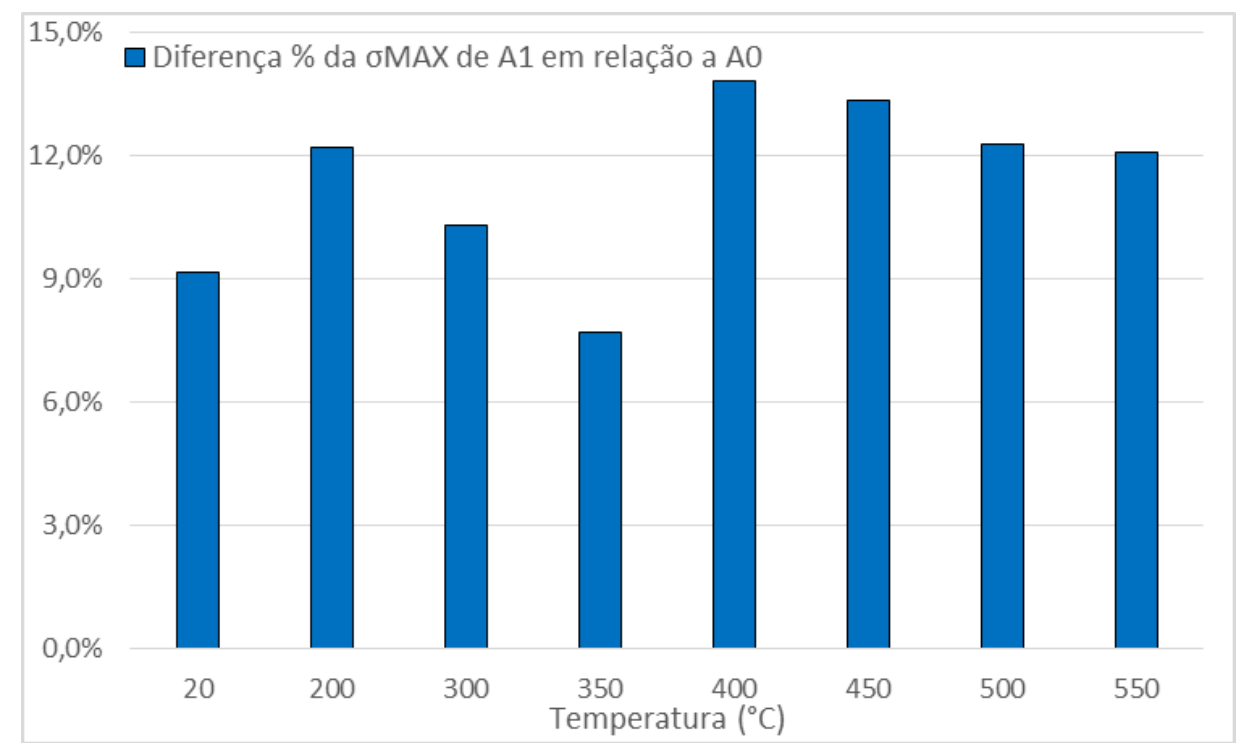

Figura 5. Variação \% do limite máximo de resistência à tração em função da temperatura de ensaio da liga $A 1$ em relação à liga $A 0$.

O resultado das análises metalográficas das duas ligas estudadas é apresentado na tabela 5, que mostra os valores do percentual de grafita/ $/ \mathrm{mm}^{2}$ para as ligas $\mathrm{A} 0$ e $\mathrm{A} 1$, tempo de exposição a quente e temperatura de avaliação. Percebe-se que com o aumento da temperatura os valores do percentual de grafita $/ \mathrm{mm}^{2}$ crescem discretamente até $300^{\circ} \mathrm{C}$. Entre $300^{\circ} \mathrm{C}$ e $350^{\circ} \mathrm{C}$ os valores caem, voltando a aumentar para temperaturas superiores a $350^{\circ} \mathrm{C}$. Acredita-se que o aumento, mais conhecido como "crescimento" da grafita, após $350^{\circ} \mathrm{C}$ tenha origem no processo de decomposição da cementita com o aumento da temperatura.

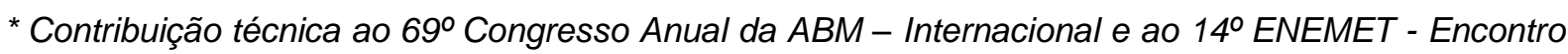
Nacional de Estudantes de Engenharia Metalúrgica, de Materiais e de Minas, 21 a 25 de julho de 2014, São Paulo, SP, Brasil. 


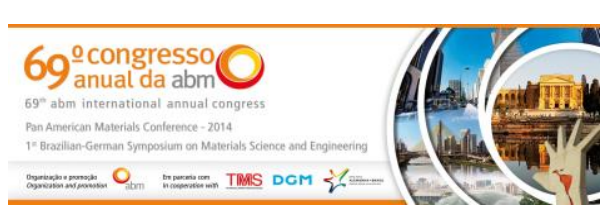

No entanto, o vale observado na figura 6 , que ilustra a curva de relação entre o percentual de grafita por milímetro quadrado e temperatura em função do tempo de exposição a quente, ainda não pode ser explicado. Também aqui, a variação do percentual de grafita entre as ligas A0 e A1 ocorre pela diferença do valor de molibdênio presente em cada um dos materiais. O molibdênio torna a matriz perlítica mais estável, dificultando o processo de decomposição da cementita. A liga A1, que possui $0,06 \%$ a mais de molibdênio do que a liga $A 0$, apresentou menores valores no aumento percentual de grafita, quando comparadas as mesmas temperaturas e tempos de exposição.

Tabela 5. Resultados da análise do percentual de grafita por milímetro quadrado para ambos os materiais expostos a diferentes temperaturas e tempos

\begin{tabular}{|c|c|c|c|c|c|}
\hline \multirow{4}{*}{$\begin{array}{c}\text { Temperatura } \\
\left({ }^{\circ} \mathrm{C}\right)\end{array}$} & \multicolumn{5}{|c|}{ Tempo de exposição a quente após homogeneização térmica } \\
\hline & \multicolumn{2}{|l|}{ Omin } & $60 \mathrm{~min}$ & $90 \mathrm{~min}$ & 120min \\
\hline & \multicolumn{5}{|c|}{$\%$ de grafita $/ \mathrm{mm}^{2}$ por material } \\
\hline & $\mathrm{AO}$ & $\mathrm{A} 1$ & A1 & A1 & A1 \\
\hline 20 & 13,44 & 9,27 & & & \\
\hline 200 & 13,70 & 9,47 & & & \\
\hline 300 & 14,18 & 10,33 & & & \\
\hline 350 & 13,55 & 8,87 & & & \\
\hline 400 & 14,18 & 11,33 & 11,42 & 11,82 & 14,41 \\
\hline 450 & 14,62 & 11,53 & 11,61 & 12,03 & 14,84 \\
\hline 500 & 15,25 & 11,88 & 12,03 & 12,39 & 15,79 \\
\hline 550 & 16,01 & 12,37 & 13,22 & 13,77 & 16,79 \\
\hline
\end{tabular}

Os tipos e tamanhos de grafita e de matriz não variaram com nenhum parâmetro de ensaio, sendo 95\%A e 5\%D para o tipo de grafita e matriz 100\% perlítica, em ambos os materiais. A distribuição do tamanho de grafita manteve-se praticamente invariável, sendo $18 \%$ do tamanho $8(0,01$ a $0,015 \mathrm{~mm}), 34 \%$ do tamanho $7(0,015$ a $0,03 \mathrm{~mm}), 28 \%$ do tamanho $6(0,03$ a $0,06 \mathrm{~mm}), 16 \%$ do tamanho $5(0,06$ a $0,12 \mathrm{~mm})$, $4 \%$ do tamanho $4(0,12$ a $0,25 \mathrm{~mm})$ e $0,09 \%$ do tamanho $3(0,25$ a $0,5 \mathrm{~mm})$.

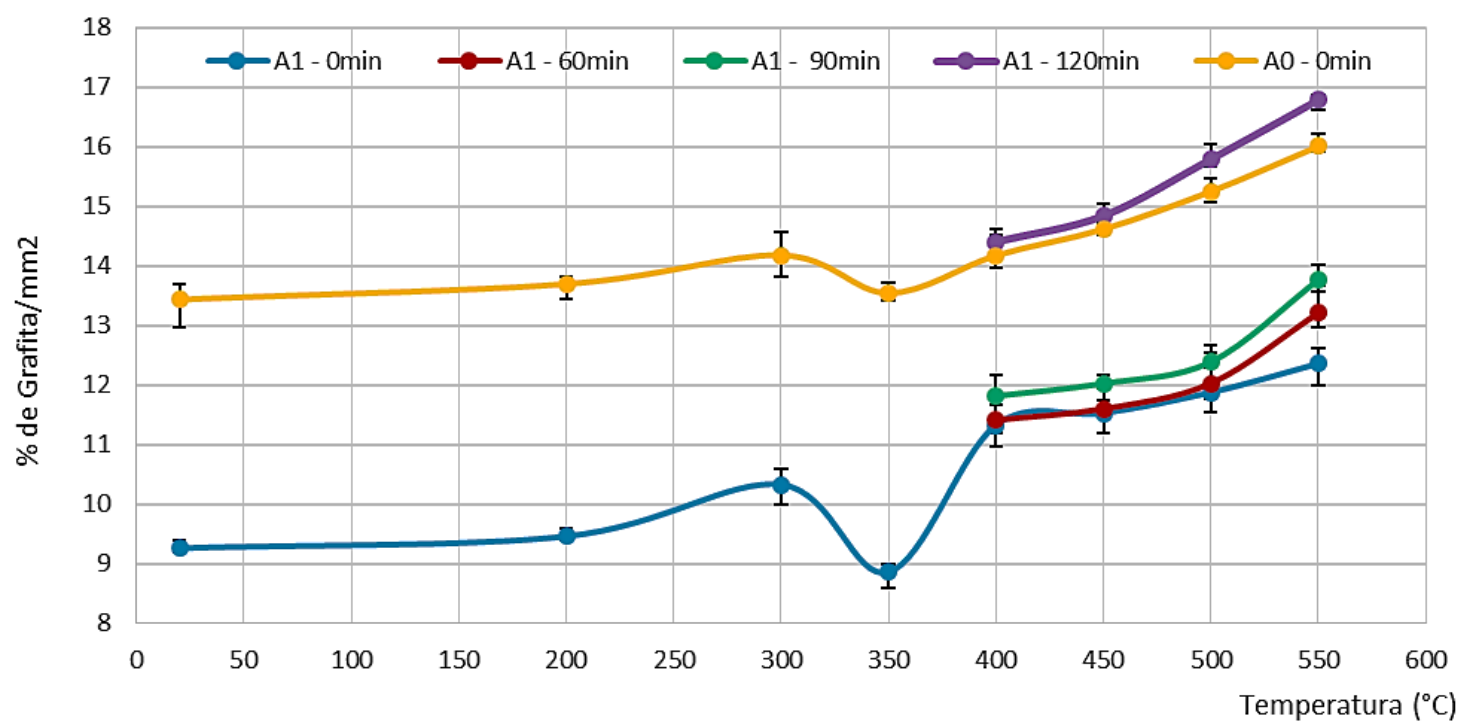

Figura 6. Curva do \% de grafita $/ \mathrm{mm}^{2}$ em função temperatura de ensaio e tempo de exposição após homogeneização térmica do material para as ligas A0 e A1.

\footnotetext{
* Contribuição técnica ao $69^{\circ}$ Congresso Anual da ABM - Internacional e ao 14ํㅡㄹ ENEMET - Encontro Nacional de Estudantes de Engenharia Metalúrgica, de Materiais e de Minas, 21 a 25 de julho de 2014, São Paulo, SP, Brasil.
} 


\section{CONCLUSÃO}

Os resultados obtidos neste estudo permitem as seguintes conclusões:

1. O molibdênio, aliado ao cromo $(0,21 \%$ em ambas as ligas) foi efetivo no sentido de minimizar os efeitos da elevada temperatura sobre as propriedades mecânicas do material em estudo.

2. O molibdênio atuou como inibidor do processo de "crescimento" das lamelas de grafita com o aumento das temperaturas, o que confirma a sua característica de estabilizador da perlita.

3. O tempo de exposição dos materiais a altas temperaturas favoreceu o processo de difusão, e sua influência ficou evidente através das análises metalográficas realizadas.

4. O teor de molibdênio influenciou no comportamento mecânico da liga a temperaturas elevadas, ocorrendo incrementos nos valores de resistência com a adição de maior quantidade deste elemento.

5. Aplicações a quente requerem uma análise detalhada de condições de trabalho. O percentual dos elementos de liga, a temperatura de trabalho e o tempo de exposição a quente podem interferir no conjunto de propriedades dos ferros fundidos cinzentos perlíticos, prejudicando assim o seu desempenho.

\section{Agradecimentos}

Os autores agradecem UDESC- Universidade do Estado de Santa Catarina, a Tupy S.A. e UNISOCIESC pela disponibilidade de suas instalações e corpo técnico para a realização das atividades experimentais e a CAPES, programa número 41002016001P9, pela bolsa de estudos concedida.

\section{REFERÊNCIAS}

1 Nechtelberger E. The Properties of Cast Iron up to $500^{\circ} \mathrm{C}$. Stonehouse: Technicopy Ltd; 1980.

2 Palmer KB. Design with cast irons at high temperatures-2: tensile, creep and rupture properties. BCIRA Journal. 1977;(25 BCIRA Report 1251):31-50.

3 Gilbert GNJ, Pidgeon CL. The growth and Scaling Properties of Cast Irons in Air at Temperatures of $350^{\circ} \mathrm{C}$ and $400^{\circ} \mathrm{C}$ for times up to 21 years. BCIRA Journal. 1982;(30): 357-394.

4 Gundlach RB. The effects of alloying elements on the elevated temperature properties of gray irons. AFS Transactions. 1983;91:389-422.

5 Turnbull GK, Wallace JF. Molybdenum effect on gray iron at elevated temperature. AFS Transactions. 1959;(67):35-46.

6 Black B, Burger G, Logan R; Perrin R, Gundlach R. A estrutura metalográfica e dimensional do GJS-SiMo a temperaturas elevadas. Fundição e Serviços.

2003;setembro:48-69.

7 Guesser WL. Propriedades Mecânicas dos Ferros Fundidos. São Paulo: Editora Blücher; 2009.

8 Gilbert GNJ. The growth and scaling characteristics of cast irons in air steam. BCIRA Journal of Research and Development. 1959;(7):478-566.

9 Bates CE. Alloy element effects on gray iron properties: part II. AFS Transactions. 1986;94:889-912.

10 Palmer KB. The mechanical and physical properties of engineering grades of cast irons up to $500^{\circ} \mathrm{C}$. BCIRA Journal. 1987; November (BCIRA Report 1717):417-425.

* Contribuição técnica ao $69^{\circ}$ Congresso Anual da ABM - Internacional e ao 14ํㅡㄹ ENEMET - Encontro Nacional de Estudantes de Engenharia Metalúrgica, de Materiais e de Minas, 21 a 25 de julho de 2014, São Paulo, SP, Brasil. 
11 Rundman KB, Rouns TN. On the effects of Molybdenum on the kinetics of secondary graphitization in quenched and tempered Ductile irons. AFS Transactions. 1982;90:487497.

12 Palmer KB. Design with cast irons at high temperatures - 1: growth and scaling. BCIRA Journal. 1976;(Report 1248):589-609.

13 Gilbert GNJ, White DG. Growth and scaling characteristics of flake and nodular graphite cast irons containing tin. BCIRA Journal. 1963;(11):295-318.

14 Kattus JR, Mcpherson B. Properties of Cast Iron at Elevated Temperatures. AFSM Publication; 1959. n.248.

15 Gundlach RB. Elevated Temperature Properties of Alloyed gray irons for diesel engine components. AFS Transactions. 1978;(86):55-64.

16 Gonçalves HC, Guesser WL. Aplicação de Ferros Fundidos Cinzentos em Elevadas Temperaturas [Trabalho de Conclusão de Curso de Especialização em Engenharia de Fundição]. Joinville: SOCIESC; 2005.

17 Röhrig K. Thermal fatigue of gray and ductile Irons. AFS Transactions. 1978;(86):75-88.

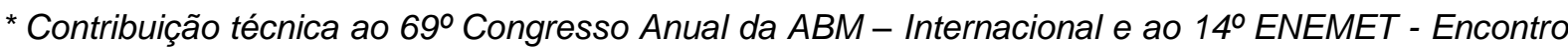
Nacional de Estudantes de Engenharia Metalúrgica, de Materiais e de Minas, 21 a 25 de julho de 2014, São Paulo, SP, Brasil. 